\title{
The PLATO Antarctic site testing observatory
}

\section{J. S. Lawrence, G. R. Allen, M. C. B. Ashley, C. Bonner, S. Bradley, et al.}

J. S. Lawrence, G. R. Allen, M. C. B. Ashley, C. Bonner, S. Bradley, X. Cui, J. R. Everett, L. Feng, X. Gong, S. Hengst, J. Hu, Z. Jiang, C. A. Kulesa, Y. Li, D. Luong-Van, A. M. Moore, C. Pennypacker, W. Qin, R. Riddle, Z. Shang, J. W. V. Storey, B. Sun, N. Suntzeff, N. F. H. Tothill, T. Travouillon, C. K. Walker, L. Wang, J. Yan, J. Yang, H. Yang, D. York, X. Yuan, X. G. Zhang, Z. Zhang, X. Zhou, Z. Zhu, "The PLATO Antarctic site testing observatory," Proc. SPIE 7012, Ground-based and Airborne Telescopes II, 701227 (10 July 2008); doi: 10.1117/12.787166

SPIE Event: SPIE Astronomical Telescopes + Instrumentation, 2008, Marseille, France 


\title{
The PLATO Antarctic site testing observatory
}

\author{
J.S. Lawrence*a, G.R. Allen ${ }^{\mathrm{b}}$, M.C.B. Ashley ${ }^{\mathrm{a}}$, C. Bonner ${ }^{\mathrm{a}}$, S. Bradley ${ }^{\mathrm{c}}$, X. Cui ${ }^{\mathrm{d}}, \mathrm{J}^{\mathrm{R}}$.R. Everett ${ }^{\mathrm{a}}$,

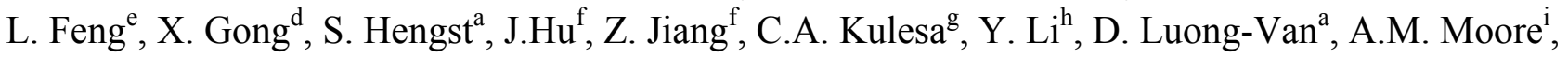 \\ C. Pennypacker, W. Qin ${ }^{\mathrm{h}}$, R. Riddle ${ }^{\mathrm{k}}$, Z. Shang ${ }^{1}$, J.W.V. Storey ${ }^{\mathrm{a}}$, B. Sun ${ }^{\mathrm{h}}$, N. Suntzeff ${ }^{\mathrm{m}}$,

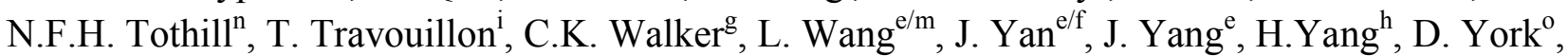

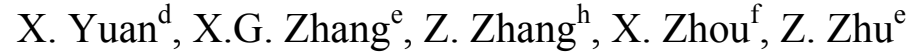 \\ ${ }^{a}$ University of New South Wales, Australia \\ ${ }^{\mathrm{b}}$ Solar Mobility, Australia \\ ${ }^{c}$ University of Auckland, NZ \\ ${ }^{\mathrm{d}}$ Nanjing Institute of Astronomical Optics Technology, China \\ ${ }^{\text {e}}$ Purple Mountain Observatory, China \\ ${ }^{\mathrm{f}}$ National Astronomical Observatory of China, China \\ ${ }^{\mathrm{g}}$ University of Arizona, USA \\ ${ }^{\text {h}}$ Polar Research Institute of China, China \\ ${ }^{\mathrm{i}}$ California Institute of Technology, USA \\ ${ }^{j}$ University of California at Berkeley, USA \\ ${ }^{k}$ Thirty Meter Telescope Observatory Corporation, USA \\ ${ }^{\text {IT } T i a n j i n ~ N o r m a l ~ U n i v e r s i t y, ~ C h i n a ~}$ \\ ${ }^{\mathrm{m}}$ Texas A\&M University, USA \\ ${ }^{\mathrm{n}}$ University of Exeter, UK \\ ${ }^{\circ}$ University of Chicago, USA
}

\begin{abstract}
Over a decade of site testing in Antarctica has shown that both South Pole and Dome C are exceptional sites for astronomy, with certain atmospheric conditions superior to those at existing mid-latitude sites. However, the highest point on the Antarctic plateau, Dome A, is expected to experience colder atmospheric temperatures, lower wind speeds, and a turbulent boundary layer that is confined closer to the ground. The Polar Research Institute of China, who were the first to visit the Dome A site in January 2005, plan to establish a permanently manned station there within the next decade. As part of this process they conducted a second expedition to Dome A, arriving via overland traverse in January 2008. This traverse involved the delivery and installation of the PLATeau Observatory (PLATO). PLATO is an automated self-powered astrophysical site testing observatory, developed by the University of New South Wales. A number of international institutions have contributed site testing instruments measuring turbulence, optical sky background, and sub-millimetre transparency. In addition, a set of science instruments are providing wide-field high time resolution optical photometry and terahertz imaging of the Galaxy. We present here an overview of the PLATO system design and instrumentation suite.
\end{abstract}

Keywords: site testing, Antarctic astronomy

* j1@phys.unsw.edu.au; phone 6129385 5003; fax 61293856060

Ground-based and Airborne Telescopes II, edited by Larry M. Stepp, Roberto Gilmozzi, Proc. of SPIE Vol. 7012, 701227, (2008) · 0277-786X/08/\$18 - doi: 10.1117/12.787166 


\section{INTRODUCTION}

Over a decade ago it was recognised that the high, dry, cold sites on the Antarctic plateau should be ideal for astronomy (see Storey ${ }^{1}$ for a recent review). Harper ${ }^{2}$ suggested that the low temperatures throughout the Antarctic plateau troposphere would result in a very low atmospheric thermal emission in the infrared. Gillingham ${ }^{3}$ predicted that the calm and stable atmosphere above the Antarctic plateau would result in weak turbulence, and that the absence of katabatic forced winds on the domes of the high plateau would result in the atmospheric turbulence being confined to a very thin but intense layer close to the surface.

These expectations motivated a series of site testing experiments (operated from 1995-2003) at the US Amundsen-Scott South Pole station $\left(0^{\circ}\right.$ east, $90^{\circ}$ south, $2835 \mathrm{~m}$ elevation). Instruments demonstrated that the infrared sky emission was 1-2 orders of magnitude lower than typically found at mid-latitude $\operatorname{sites}^{4-7}$, and that the atmosphere held extremely low precipitable water vapour ${ }^{8}$. The expectation of weak free-atmosphere turbulence was also confirmed. However, the very strong turbulent surface layer 200-300 metres thick was found, resulting in mediocre ground level seeing ${ }^{9-11}$.

The Italian/French Concordia station at Dome C (123 east, $75^{\circ}$ south, $3250 \mathrm{~m}$ elevation), lying at a higher altitude than South Pole, was expected to experience colder atmospheric temperatures, resulting in even lower thermal emission and lower water vapour content. Additionally, the local topography of Dome $\mathrm{C}$ indicated that the surface wind speeds should be lower, and thus the turbulent boundary layer should be confined closer to the surface ${ }^{2,12}$. These expectations have been confirmed by a series of site testing experiments operated over the last 5 years. The sub-millimetre opacity and infrared atmospheric emission was shown to be lower than observed at South Pole ${ }^{13,14}$, and while the wintertime surface boundary layer was also found to exhibit relatively strong turbulence, this was confined to within $\sim 30 \mathrm{~m}$ of the surface ${ }^{15-17}$.

Dome A ( $74^{\circ}$ east, $71^{\circ}$ south, $4100 \mathrm{~m}$ elevation) lies at the highest point of the Antarctic plateau. Regional meteorological climate models predict that the near-surface wind speeds at Dome A should be weaker than anywhere else on the plateau ${ }^{18}$. These predictions have been confirmed by Automated Weather Station data from the site ${ }^{19}$. Wind shears within the near-surface layer cause turbulence; the near-ground turbulence at Dome A is expected to be confined to a much thinner layer than other high plateau sites ${ }^{20}$. The higher altitude of Dome A is also expected to result in a significantly colder and drier atmosphere than other plateau sites ${ }^{21}$. PLATO (PLATeau Observatory), an autonomous self-powered observatory with a suite of astronomical site testing instrumentation, was developed to quantify these atmospheric characteristics. PLATO was deployed to the Dome A site in early 2008, as part of the International Polar Year PANDA expedition conducted by the Polar Research Institute of China and the Chinese Academy of Sciences.

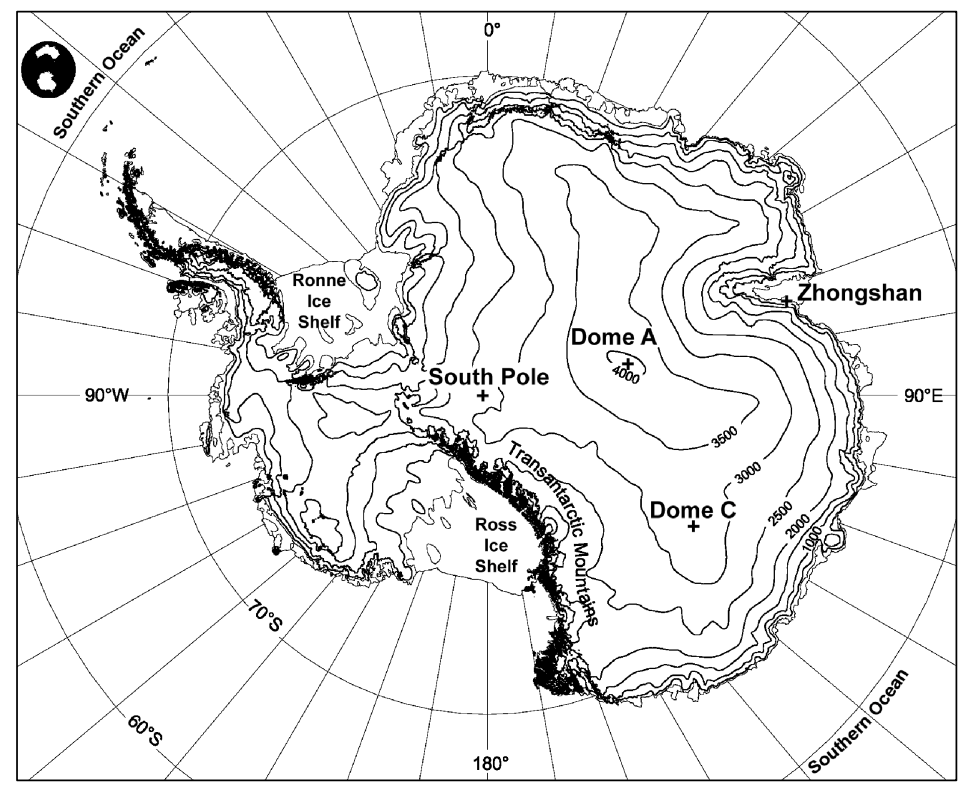

Fig. 1. Map of Antarctica showing the Chinese coastal station Zhongshan and the high plateau stations South Pole, Dome $\mathrm{C}$ and Dome A. Basic map courtesy of the Australian Antarctic Data Centre. 


\section{THE PLATO OBSERVATORY}

PLATO is a modular observatory that was designed to provide continuous power and heat, and a robust command, control, and communications system for a range of site testing instruments. As there is currently no infrastructure at Dome A, the main design requirement for PLATO was that it be completely self-supporting, i.e., generating its own heat and electricity, and being able to operate robotically with only minimal external control via a low-bandwidth satellite communication link. It was also required to operate in the extreme Dome A environmental conditions: low ambient temperature range (from $-30^{\circ} \mathrm{C}$ in summer to $-90^{\circ} \mathrm{C}$ in mid-winter), at low atmospheric pressure ( 570 mbar), with high relative humidity (typically 50\% with respect to the dew-point but super-saturated with respect to the frost-point), and a 130 day continuous period of darkness.

The PLATO observatory was designed and constructed during 2006-2007 at the University of New South Wales. Instruments contributed by various international teams were integrated into the observatory in late 2007. PLATO was then shipped to Fremantle where it was loaded onto the Polar Research Institute of China (PRIC) icebreaker "Xuelong". After a two week voyage it arrived at the Chinese Antarctic coastal station Zhongshan, where the Dome A expedition was assembled. The PRIC expedition, with scientific goals in fields including astronomy, glaciology, sub-glacial geology, climatology and upper atmospheric physics, consisted of a 17 -member team with a range of scientific equipment, transported by an over-ice tractor convoy. The traverse arrived at Dome A on 11 January 2008, after a $\sim 1300 \mathrm{~km}$ journey from the coast taking 22 days. The expedition spent 15 days at the Dome A site, during which time PLATO and its instrumentation suite were assembled and tested, before the team returned to the coast. At the time of writing (May 2008), all PLATO systems are still operational, having run unmanned for over 120 days.

PLATO consists of two separate modules: the engine module, which houses the primary power source, and the instrument module, which houses the power electronics, and control and communication systems. Solar panels are mounted externally. Instruments are mounted either on the roof (with power and control cables connected through a series of portholes) or externally on the snow surface. The two modules are each built into a standard 10 foot shipping container, and are designed to be coupled to each other and shipped as an ISO standard 20 foot container. This allows for convenient transport via road, rail, sea, and ice sled. The modules are both heavily insulated, using 150-200 mm thick polyurethane-foam/colourbond-steel sandwich panels designed for a module heat loss of 10-15 W/K. Measurements at Dome A during summer and winter have now confirmed this thermal performance.

PLATO was designed based on a power budget for required average/peak power load of 800/1600 W during winter, dropping to $400 / 1200 \mathrm{~W}$ during the summer months when several instruments requiring dark skies are not operational. This power is sufficient to maintain the engine module temperature well above $0^{\circ} \mathrm{C}$ using waste engine heat alone. A closed-loop PID temperature controller is used to regulate the engine module temperature to around $15^{\circ} \mathrm{C}$ by extracting hot air. This set-point allows a long thermal time constant in the event of an engine failure, but also ensures the fuel is well above freezing point. The instrument module is electrically heated, mostly from instruments and electronic components within the module, but with the option of switching in additional resistive heaters when required. This is generally sufficient to maintain an internal temperature in the range $-20^{\circ} \mathrm{C}$ to $0^{\circ} \mathrm{C}$, with external ambient temperatures as low as $-80^{\circ} \mathrm{C}$. Several air circulation fans are also installed to prevent large temperature gradients due to stratification. The critical electronic components in the instrument module have been tested for low temperature operation and have been found to operate satisfactorily at temperatures well below the conservative lower limit set-point for this module.

\section{PLATO POWER GENERATION}

The PLATO power system is described in a separate paper ${ }^{22}$, so only a brief summary is given here. The primary power source for PLATO is a set of six Hatz 1B30 diesel engines. These are housed in the engine module, which also contains a 4000 litre fuel tank and engine control electronics. The Hatz 1B30 is a compact, high efficiency, single-cylinder aircooled diesel engine of $350 \mathrm{cc}$ displacement. The engine was tested for starting behaviour, efficiency, and thermal behaviour in a purpose-built low pressure test chamber at the University of New South Wales. These tests demonstrated a maximum power output of greater than $1.5 \mathrm{~kW}$ at atmospheric pressure corresponding to those measured at Dome A, and a pressure-independent efficiency of 350-400 $\mathrm{g} / \mathrm{kWhr}$ (at the applicable operating parameters for PLATO). The engines are run on Jet-A1 aviation fuel, a choice motivated by the ready availability of this fuel in Antarctica and its low freezing point. Lubricity modifiers were added to extend engine life. A bank of large fuel filters ensures a clean fuel 


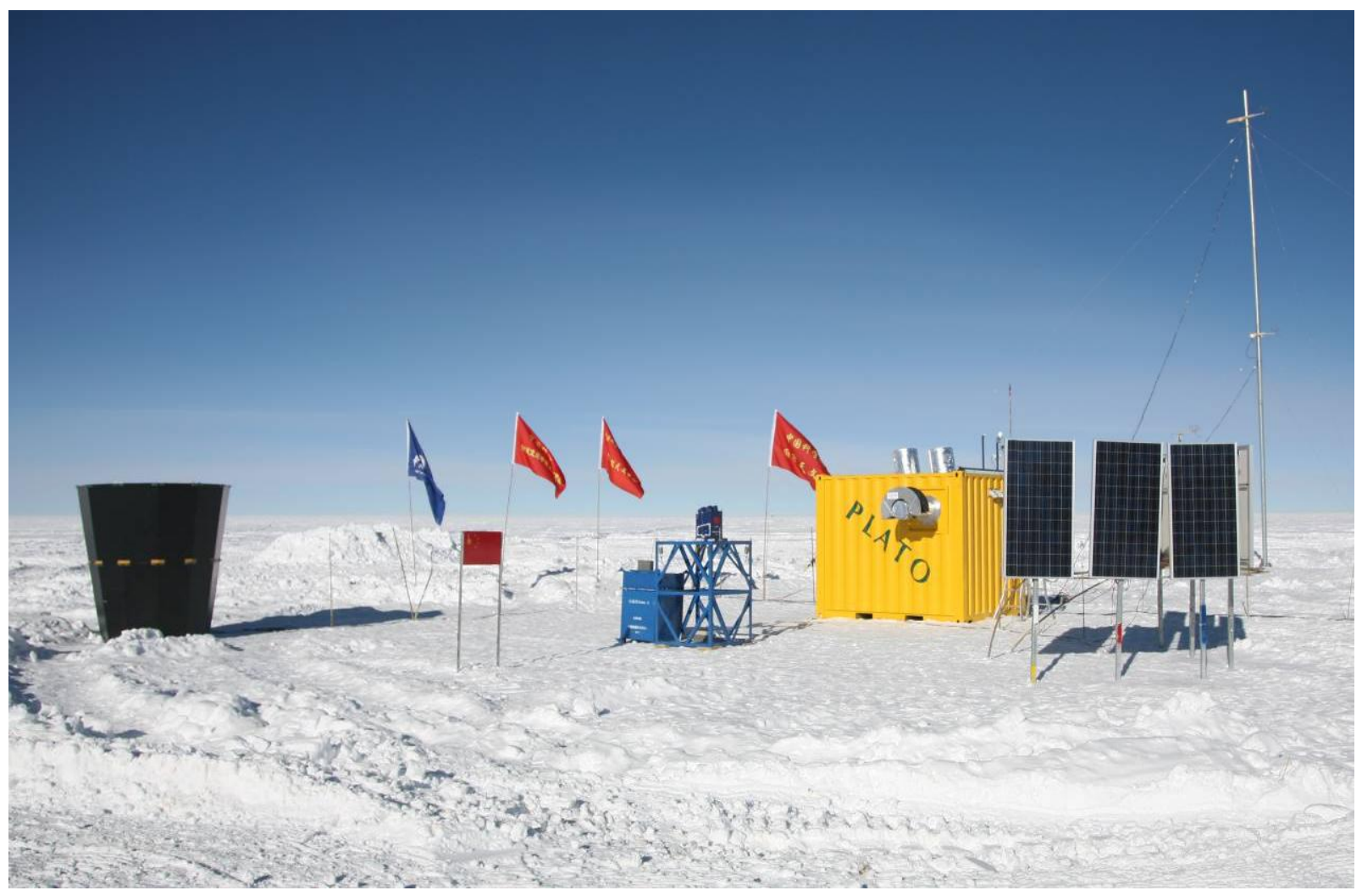

Fig. 2. Picture of the PLATO observatory installation at Dome A station. The PLATO instrument module is centre right surrounded by the solar panel array, the CSTAR telescopes (centre) and the SNODAR instrument (left). Image courtesy Zhou Xu, Zhenxi Zhu.

supply for a minimum of one year between servicing. The fuel tank contains enough capacity for greater than nine months of continuous running and is also used to store heat from the engines to help regulate the internal temperature of the module. In addition, each engine is installed with its own bulk oil filtration and recirculation system in order to extend the required servicing interval from the nominal 200 hours of a stock engine, to the $\sim 2000$ hours we require.

The engines are run at a fixed speed of about 2200 RPM. The low running speed is optimal for fuel consumption and wear, while the constant running speed allows for vibration control through an appropriate choice of dampers. Each engine has its own generator that produces 120-150 VDC. The engines are arranged in two banks of three for redundancy, and two separate high voltage power cables are connected back to the instrument module. Engine start power is supplied by ultra-capacitor banks (one for each engine bank). The ultra-capacitor array is used in a similar way to a lead acid battery, but operates to temperatures below $-40^{\circ} \mathrm{C}$ and provides ideal charging behaviour and extremely high discharge current capacity. The two 12 VDC arrays have capacitances of 500 and $1000 \mathrm{~F}$ respectively, taking 10-20 minutes to fully charge from the PLATO battery bank, and allowing more than 20 start attempts before requiring recharge. In practice, the ultra-capacitors have been highly successful in this role.

During periods when there is sun available, up to $1 \mathrm{~kW}$ of electrical power is generated from a solar panel array. This power is used to reduce total fuel consumption, and is sufficient to power the entire observatory during the summer months in the event of an engine system failure. There are two arrays of three vertically mounted mono-crystalline panels (nominally rated at $167 \mathrm{~W}$ ) that face North-East and North-West. This orientation is designed to maximise power when the sun last sets (in early April) and first rises (in late August). The high reflectivity of the ice and the low ambient temperatures allow for high light gathering and conversion efficiency, with each panel producing a maximum of over 
$200 \mathrm{~W}$, and an average of $\sim 2 \mathrm{kWhr} /$ day when the sun is above the horizon for greater than $\sim 12$ hours. Total solar power over 1 year is expected to be $\sim 2000 \mathrm{kWhrs,} \mathrm{representing} \mathrm{a} \mathrm{fuel} \mathrm{saving} \mathrm{of} \sim 1000$ litres.

All PLATO systems are powered from a 24 VDC 320 Ahr battery bank, consisting of six $4 \mathrm{~V}$ gel cells. This provides uninterrupted power to the instruments, and allows for multiple engine restart attempts if required. The battery bank is charged by the diesel engine array and the solar panel array. The 120-150 VDC output from the engine banks is regulated to the appropriate temperature-dependent battery float voltage using two sets of parallel pair DC/DC switch mode converters, with a set-point controlled by the PLATO supervisor computer. The nominally 50 VDC output from the solar panels is regulated to the appropriate battery float charging voltage by a commercial solar panel controller, one for each array. This unit also performs maximum power-point tracking, for the most efficient use of the solar panels.

Engine control and monitoring is via the PLATO Controller Area Network (CAN) system bus. Cylinder head temperature, exhaust temperature, oil temperature, and oil pressure are measured for each engine, and automatically logged every 60 seconds. Control is via 3 digital switches to each engine: start (starter motor solenoid), stop (fuel solenoid valve), and warm (air intake glow plug). Additionally, the fuel pump rate and the oil recirculation pump rate can be controlled separately for each engine. Only one engine is run at any time in normal operation. A second engine is automatically started when an engine's health is low, has had an extended run time, or when excess power is required. Scripts running on the two redundant "supervisor" computers in PLATO control the engines, and automatically restart them as required.

\section{PLATO CONTROL}

The PLATO power system is described in a separate paper ${ }^{23}$, so only a brief summary is given here. PLATO is designed to run unattended for periods of up to one year. To achieve this level of autonomy, multiply-redundant Linux-based "supervisor" computers, each with their own management electronics and satellite modem, provide multiple paths of communication with each other and the outside world. The supervisor computers monitor and control the PLATO power distribution, thermal, and engine management subsystems via a CAN (Control Area Network) bus network. Highbandwidth communication between the instruments and the supervisor computers is provided via a $100 \mathrm{Mbit} / \mathrm{s}$ wired LAN. Communication with the module is via the Iridium satellite network.

PLATO has two supervisor computer nodes, each in a thermally regulated (19-inch rack mount) enclosure. Each node consists of a PC104 computer stack, a CAN microcontroller interface board, a general purpose interface (GPI) PCB, and an Iridium L-band transceiver. Each PC104 computer stack consists of a processor module (Parvus \#CPU-1452 400 $\mathrm{MHz}$ Celeron with $256 \mathrm{MB}$ SDRAM), a power supply module, and a series of 4-8 GB USB flash drives. The USB flash drives were selected after testing the low temperature operation of a range of commercial devices. A Sandisk 4 GB drive rated to $-25^{\circ} \mathrm{C}$ was found to work reliably to $-55^{\circ} \mathrm{C}$. Each drive is partitioned into a series of logical partitions that are mounted read-only except when data is actually being written to the drive. This minimises the chance of file system corruption destroying large blocks of data. The CAN microcontroller interface board communicates between the supervisor via RS485 and the local CAN network. The GPI PCB board is a custom-built board for power switching (thermal control and iridium power), analog input/output (for thermal regulation), and watchdog timing. The iridium Lband transceiver communicates with the PC104 via RS232, and is connected to an externally mounted low temperature antenna.

The supervisor computer real-time clock and CMOS power is maintained using two D-cell lithium thionyl chloride batteries, with a switched-mode voltage regulator, which insures that the voltage remains within specification down to at least $-60^{\circ} \mathrm{C}$. The computer BIOS settings are such that the computer boots correctly even if power is lost to the CMOS.

The PLATO CAN network consists of a series of units, in both modules, for power distribution, thermal regulation, and engine control. Power distribution units (located in the instrument module) provide the capability for switching (via a series of ESD protected high-side switches) and current monitoring (via current shunt resistors) for up to 16 channels, with hardwired self-resetting solid-state fuses current limiting each channel pair to $12 \mathrm{~A}$. These units are used for instrument and PLATO component power. Thermal control units (located in each module) provide communication between the PID controllers and the CAN bus, provide power for the instrument module heaters and air circulation fans, and monitor temperature with a series of inputs for (AD590) 2-terminal IC temperature transducers. Engine control units (located in the engine module) contain a series of analog/digital inputs for engine monitoring and a series of high side switches for engine control and engine ancillary power. 
The supervisor computers run a Debian etch GNU/Linux operating system with a read-only root filesystem on a USB flash drive. This has proven to be extremely reliable - the computers can have their power interrupted without danger of file-system corruption. Each computer transfers data to/from the internet using the Iridium modems, both using direct internet connection via PPP to the Iridium groundstation, and with Short Burst Data (SBD) messages. In practice, we can maintain an average bandwidth of around 280 bytes per second per modem, which is sufficient for command-andcontrol, health-and-status data, as well as returning a subset of reduced data from the experiments. Data is automatically uploaded to various webpages for human inspection, and SMS messages can be automatically sent to mobile phones to inform a human that manual intervention is required. It is possible to establish an ssh link to PLATO at any time within a few minutes. The individual instrument Principal Investigators can have two-way communication with their own experiments via Iridium SBD messages.

\section{PLATO INSTRUMENT SUITE}

The PLATO system is designed to provide power, heat, and communication to a range of site testing and science instruments. Each of the instruments is designed to be as simple as possible, with few moving parts. See Ashley et al. ${ }^{24}$ and Strassmeier et al. $2007^{25}$ for a discussion of specific requirements for Antarctic instrumentation. The instruments each have their own independent computer, data storage systems, and automated operating scripts, and communicate with the PLATO supervisor computers via a local area network. Each instrument is allocated a budget for peak power and 24 hour average power, which it is responsible for distributing between its own various instrument and heating functions. Instruments are mounted either externally on the snow surface, externally on the PLATO instrument module roof ports, or partially internal/external to the instrument module (i.e., through wall or roof ports).

Key atmospheric properties measured by PLATO include the structure of the surface-layer atmospheric turbulence above the site (Snodar and DASLE), the optical sky background and cloud cover (Gattini and webcams), and the atmospheric transparency and emission at sub-millimetre wavelengths (Pre-HEAT). Scientific fields covered by PLATO instruments include optical transient astronomy (CSTAR) and mapping of the Galactic plane (Pre-HEAT).

\subsection{CSTAR}

CSTAR (Chinese Small Telescope ARray) is composed of four telescopes with a fixed orientation, each with a different optical filter, observing a $4.5^{\circ} \times 4.5^{\circ}$ field centred on the South Celestial Pole. Each telescope takes an image every 1030 seconds throughout the Antarctic winter period. Such a high cadence on a single wide field, with multi-colour photometry, will provide a unique dataset. There are a range of scientific objectives for this telescope in several fields of variable and transient astronomy. These include supernovae studies, gamma-ray burst optical afterglow detection, exoplanet detection (through transit and microlensing techniques), and variable star light curves and statistics. This dataset will additionally provide site characterization of the optical sky background, cloud cover, and aurora.

The CSTAR design is particularly suited to Antarctic operation, having no moving parts. The instrument is installed external to PLATO on the snow surface using a tripod mount. The telescopes are identical Schmidt-Cassegrain designs with a focal ratio of $\mathrm{f} / 1.2$. Each has a $145 \mathrm{~mm}$ entrance aperture and a $1024 \times 1024$ (Andor DV435) frame transfer CCD array, sampled at $16 \operatorname{arcsec}$ per $13 \mu \mathrm{m}$ pixel. Three of the telescopes have a fixed filter, providing imaging in $\mathrm{g}, \mathrm{r}$, and $\mathrm{i}$ bands, the fourth telescope is filter-less. While the CCDs each have a Peltier cooling capability, it is intended that they operate passively, i.e., at the external ambient temperature. Laboratory tests have confirmed operation as low as $-80^{\circ} \mathrm{C}$. The telescopes are installed in a hermetically sealed enclosure and have an indium-tin-oxide coating applied to the front windows. This conductive coating provides approx $10 \mathrm{~W}$ of heating per camera, which has proven to be very successful in avoiding snow and ice formation on the external windows. Each camera has its own industrial computer installed inside the PLATO instrument module. Each computer uses a compact flash hard drive for the operating system and camera control software, and a 750 GB USB hard drive for data storage. Data collection, storage, and preliminary photometric analysis software for CSTAR is fully automated. While the majority of data will be retrieved during the next expedition (in early 2009), a subset of images and reduced data is returned each day via the PLATO Iridium network allowing nominal operation of the instrument to be confirmed. 


\subsection{Pre-HEAT}

Pre-HEAT is a $0.2 \mathrm{~m}$ aperture sub-millimetre wave "tipping" telescope with a heterodyne receiver ${ }^{26}$. Pre-HEAT was designed principally to measure the atmospheric opacity at $450 \mu \mathrm{m}$; this is derived from a measurement of the atmospheric emission at this wavelength as a function of zenith angle. Such a measurement allows the total atmospheric precipitable water vapour content to be determined, which has important implications for observations at wavelengths from the sub-millimetre to the near infrared. Pre-HEAT will also obtain the first astronomical spectra from the Dome A site. It will construct strip maps of ${ }^{13} \mathrm{CO} \mathrm{J}=6-5$ line emission in the Galactic plane (at $10 \mathrm{arcmin}$ resolution). This molecule is an important tracer of the dark, cold gas and dust clouds in the interstellar medium. Additionally, Pre-HEAT acts as the technological forerunner for HEAT, the High Elevation Antarctic Telescope, a $0.6 \mathrm{~m}$ aperture terahertz telescope proposed for future deployment to Dome A.

Pre-HEAT is designed as a light weight, low power, fully autonomous instrument. It consists of a $0.2 \mathrm{~m} \mathrm{f} / 5$ parabolic mirror at the end of an enclosed telescope assembly tube which mounts through a port in the PLATO side wall. The mirror scans the sky in elevation and has a co-rotating external (teflon) window. The receiver, a $660 \mathrm{GHz}$ Schottky diode system, is mounted inside PLATO on a sensor assembly unit which also houses the IF processor, IF power detector, and digital FFT spectrometer. Pre-HEAT uses a PC104 single board computer with 8 GB flash disc hard drives.
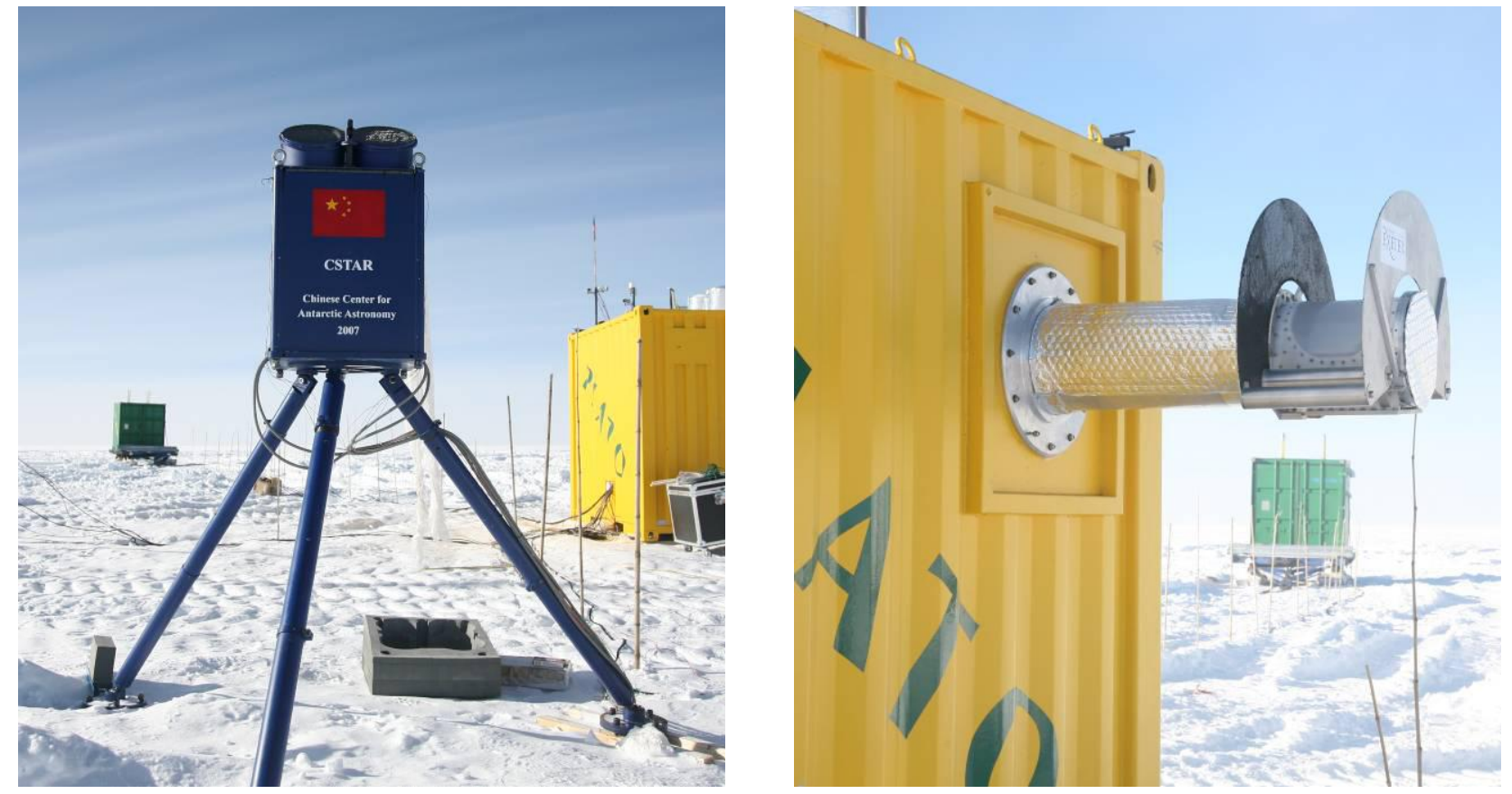

Fig. 3. Left: the CSTAR optical telescope array tripod-mounted on the snow surface. Right: the Pre-HEAT sub-millimetre telescope mounted through the PLATO instrument module side wall. The PLATO engine module is located in the background of both pictures. Images courtesy Zhou Xu, Zhenxi Zhu.

\subsection{Snodar}

Snodar (Surface layer NOn-Doppler Acoustic Radar) was designed to measure the height and intensity of the atmospheric boundary layer turbulence on the high Antarctic plateau ${ }^{27}$. It is based on the SODAR (SOund Detection And Ranging) technique, which works by sending an intense acoustic pulse into the atmosphere and listening for backscatter. The pulse is scattered by refractive index inhomogeneities, which are caused by small-scale turbulent temperature fluctuations arising from the combination of temperature gradient and wind shear. Snodar operates in monostatic mode, i.e., it has a co-located vertical-pointing source and receiver. It is designed to measure the backscattered acoustic intensity, which is proportional to the atmospheric temperature structure function, hence the refractive index structure function and astronomical "seeing". In contrast, commercial SODARs also typically measure wind speed via the Doppler shift of the reflected signal. 
At the surface temperature range $\left(-30^{\circ}\right.$ to $\left.-80^{\circ} \mathrm{C}\right)$ and relative humidity $(>50 \%)$ experienced at Dome A, atmospheric acoustic absorption is both stable (with respect to temperature and humidity changes) and low. This allows (relative to STP conditions), a more accurate calibration, and either a longer range for a given frequency or a higher frequency (giving better vertical resolution) for a fixed range. Snodar has thus been designed specifically for high sensitivity and resolution in the near surface layer.

Snodar operates between $3 \mathrm{kHz}$ and $14 \mathrm{kHz}$. It uses a single horn-loaded compression driver as both transmitter and receiver, and a $1.5 \mathrm{~m}$ off-axis parabolic reflector to collimate the acoustic beam. It has a vertical resolution of $1 \mathrm{~m}$ and a minimum sampling height of just $8 \mathrm{~m}$. The parabolic reflector and acoustic transducer are heated and housed in a $2.4 \mathrm{~m}$ tall flat-packable sound-cone lined with an acoustic absorbing material. A calibration sphere is mounted at a height of $1.7 \mathrm{~m}$ above the dish. The instrument is located on the snow surface $\sim 30 \mathrm{~m}$ away from PLATO. The Snodar control electronics are located in an instrument rack inside the PLATO instrument module. A dedicated PC/104 computer performs all signal processing in real time and uses a USB soundcard and audio amplifier for analogue signal I/O. Snodar runs autonomously, storing raw data on USB flash disks, and sending processed data via the PLATO system Iridium satellite network link through the PLATO supervisor computer. This link also allows for Snodar operating scripts to be remotely configured.

\subsection{Gattini}

The percentage and duration of dark time for optical astronomy is a critical value for ascertaining the quality of Dome A as a site for a future optical observatory. The Gattini instrument consists of two cameras for the measurement of optical sky brightness, detection of bright aurora, cloud cover, and variability of airglow of the winter sky above Dome A. The cameras are located on the roof of PLATO and point towards the celestial South Pole, taking images of the night sky every few minutes. One camera, called "Gattini All Sky", takes wide field images using Bessel B, V and R filters including a long pass red filter for the detection of airglow. The second camera, called "Gattini-SBC", has a larger aperture and narrower field of view and will monitor the sky brightness in a range of the Sloan survey filter bands ( $\mathrm{g}^{\text {', }} \mathrm{r}$ ' and $\left.\mathrm{i}^{\prime}\right)$. The Gattini Dome A cameras are an upgraded version of a similar instrument currently operating at Dome $\mathrm{C}^{28}$.

Both cameras are mounted in thermally insulated enclosures fixed to the PLATO roof ports that are regulated to a set point of $-15^{\circ} \mathrm{C}$, and include internal fans and heaters to avoid ice formation on the external and internal windows. Both cameras use a commercial $2048 \times 2048$ pixel USB interline CCD array (Apogee U4000) with $7.4 \mu \mathrm{m}$ square pixels. The arrays are Peltier cooled to a set point of $-60^{\circ} \mathrm{C}$, sufficient for negligible dark noise. The SBC camera lens has a focal length of $300 \mathrm{~mm}$ giving a FOV of $2.8^{\circ} \times 2.8^{\circ}$ with a plate scale of $\sim 5 \operatorname{arcsec} /$ pixel. The all-sky camera uses a lens of focal length $10.5 \mathrm{~mm}$ giving a field of view $80.9^{\circ} \times 80.9^{\circ}$.

The Gattini (and DASLE) control system is a Linux-based computer system housed in the PLATO instrument module. The control of all instrumentation is accomplished through a custom software suite. This software controls all aspects of how the instruments gather data, switch between operational modes, and correct for errors in the operations of the subsystems. Data archiving is accomplished daily; the software recognizes errors in the hard drive operation, and can switch between hard disks if one dies.

\subsection{DASLE}

DASLE (Dome A Surface Layer Experiment) studies the meteorological conditions in the atmospheric boundary layer. These measurements are critical to the site evaluation of Dome A. Studies at South Pole and Dome C have shown that a strong temperature inversion exists during winter, and wind-shear within this creates most of the turbulence responsible for the image degradation of the telescopes. DASLE will measure the intensity and vertical extent of the boundary layer using three fast sonic anemometers. These instruments, manufactured by Apptech and modified for the Antarctic environment, measure temperature and 3D wind velocity at $200 \mathrm{~Hz}$ from which the turbulence can be deduced ${ }^{29}$. Three sensors are installed along a $15 \mathrm{~m}$ tower. This will be extended to $30 \mathrm{~m}$ in the next traverse. The data from the three anemometers is synchronised electronically, recording the 3 wind components and temperature at a rate of $20 \mathrm{~Hz}$ after being processed internally from the $200 \mathrm{~Hz}$ raw data used to compensate for aliasing, temporal and spatial averaging. Each day, the data is automatically compressed and backed up, and a small sample of health and status data is sent via the PLATO supervisor computer.

The instrument's management is handled both electronically and through software. Like other metal structures, the anemometers are prone to ice formation. To remedy this problem, the anemometers are periodically switched off and 
heated by a resistance tape wrapped around the shaft and the transducer. Thanks to our experience with the same instruments deployed at Dome $\mathrm{C}$ for the past 2 winters $^{30}$, it was determined that a heating cycle of 20 min every hour was adequately keeping the instrument ice free while maximizing the amount of data it can gather. In order to minimise the peak consumption of the instrument, each anemometer is heated in turn.

\subsection{Webcams}

A set of four 4 low-power CCD webcams are mounted on the PLATO roof. These are designed primarily for housekeeping purposes, i.e., to check on the snow and ice accumulation on instruments and surfaces and to monitor engine module exhaust streams. They also provide valuable qualitative assessment of various aspects of the site conditions. For example, they allow rough estimates of the cloud cover (by direct detection if the sun or moon is up, else by the presence/absence of stars), give a visual record of wind strength and direction (through the exhaust stream from the engines), and have detected several aurora in the night sky.

The PLATO webcams use various models of Watec camera: three are low-light level monochrome, and one is colour. Each uses a $1 / 2$ inch interline transfer CCD with approximately $800 \times 600$ pixels. They have different lenses (with 4-12 $\mathrm{mm}$ focal lengths) giving fields of view ranging from $30^{\circ} \times 23^{\circ}$ to $88^{\circ} \times 66^{\circ}$. Each camera has a composite video output which is digitised via a 4-port video server connected to the PLATO local area network. Images are collected from each camera every 5 minutes.
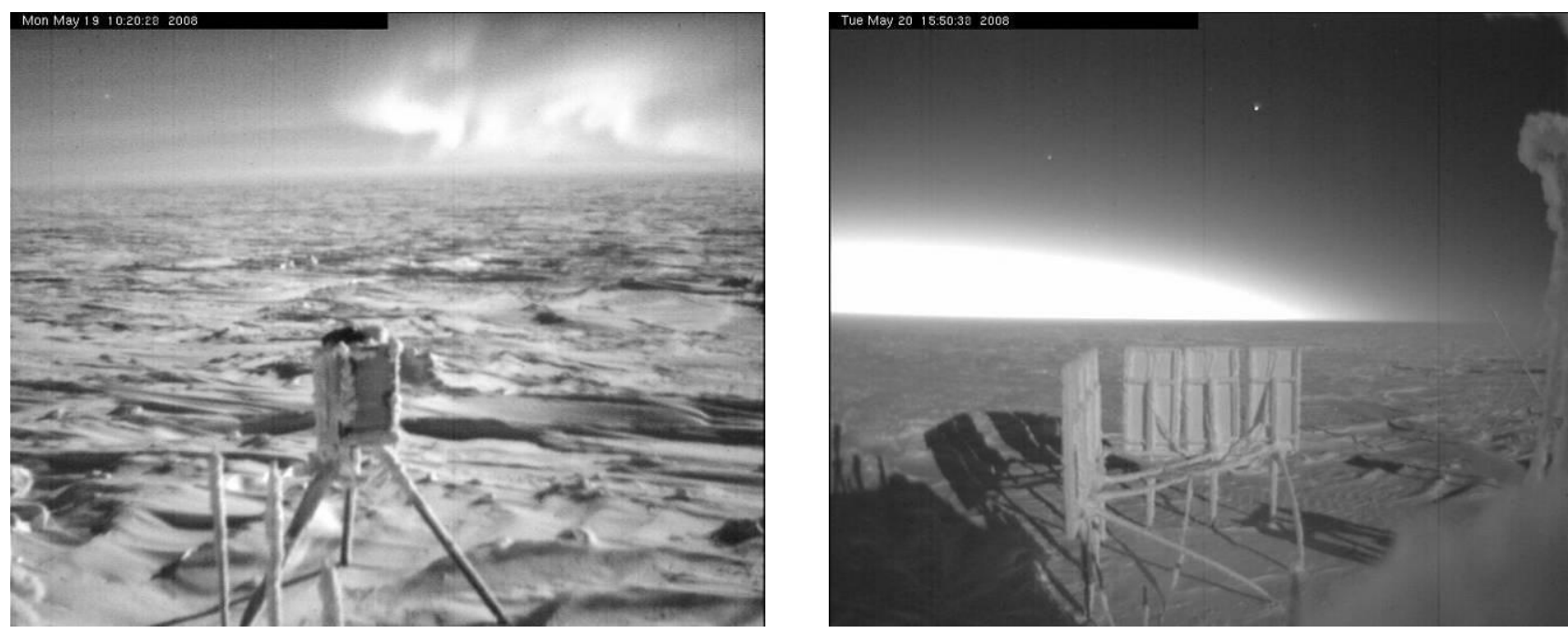

Fig. 4. Examples of webcam images from Dome A taken during winter, and retrieved via Iridium satellite link. The left picture, taken on 19 May 2008, shows the CSTAR telescope in the foreground, with auroral activity close to the horizon. The right picture, taken on 20 May 2008, shows the solar panel array in the foreground, illuminated by moonlight.

\section{FUTURE PLANS}

\subsection{Future site testing instruments}

The Polar Research Institute of China intends to return to the Dome A site each summer over the next few seasons, with the aim of setting up a permanently manned base within the next decade. As part of these future expeditions a number of upgrades to the PLATO system are planned, including the deployment of a series of second generation site testing instruments. These include Nigel, Lunar SHABAR, MASS, and PIE.

The Nigel instrument consists of a fibre-fed optical spectrometer designed to measure the optical sky spectrum at various elevation angles ${ }^{31}$. This instrument aims to determine the magnitude and spatial and temporal distribution of aurora above Dome A. As Dome A lies within the auroral oval, aurora are likely to contribute significantly to the optical sky brightness $^{32}$. The lunar SHAdow Band And Ranging (SHABAR) instrument employs an array of photodiodes which measure the variation of lunar intensity over time. From these scintillation statistics the profile of night-time turbulence 
within the ground layer can be derived ${ }^{33}$. MASS (Multi-Aperture Scintillation Sensor) measures the high-altitude turbulence with low spatial resolution but high temporal resolution (see Kornilov et al. ${ }^{34}$ ). The Dome A MASS, currently under development, is a modified version of a gimbal-fed refractive telescope system that was previously deployed to Dome $\mathrm{C}^{35}$. PIE (PLATO Icing Experiment) is an experiment designed to investigate characteristics of ice and frost formation on various surfaces in the supersaturated near surface layer air at Dome A, and techniques for avoiding such ice formation on optical surfaces and instrument windows. PIE will benefit from an earlier experiment, Givre ${ }^{36}$, operated over the last few seasons at Dome C.

\subsection{Future facilities}

Recent site-testing results have generated significant interest in Antarctic locations for astronomy. Currently there are several facilities being proposed at various sites. At the US Amundsen-Scott South Pole station the $10 \mathrm{~m}$ diameter millimetre and sub-millimetre wave South Pole Telescope recently (in 2007) obtained first light. At the French/Italian Dome C station, facilities such as the $2.4 \mathrm{~m}$ optical/infrared PILOT (the Pathfinder for an International Large Optical Telescope), and the $12 \mathrm{~m}$ diameter submillimetre wave ASO (Antarctic Submillimetre Observatory) telescope are now being considered, as successors to the $0.8 \mathrm{~m}$ IRAIT infrared telescope ${ }^{37}$.

For Dome A, there are several proposals for the next generation of astronomical facilities. These include HEAT (High Elevation Antarctic Telescope), AST3 (Antarctic Schmidt Telescope), and Xian. HEAT is a $0.6 \mathrm{~m}$ terahertz telescope observing at $0.8,1.5$ and $1.9 \mathrm{THz}(370,205$ and $160 \mu \mathrm{m})$. It is the successor to PLATO's Pre-HEAT instrument. The key science goals for HEAT are to understand molecular cloud formation in the Galaxy by performing large scale mapping surveys at the carbon, nitrogen, and oxygen fine structure and molecular lines ${ }^{38}$. AST3 is a successor to the CSTAR instrument; it consists of an array of three $0.5 \mathrm{~m}$ wide-field (10 square degree) Schmidt telescopes with large format CCD arrays. It is designed to obtain light curves for a large sample of Type 1a Supernovae, and to search for exoplanets through gravitational microlensing. Xian is an ambitious facility proposed to follow on from AST3. It consists of an array of up to 400 Schmidt telescopes, each fixed in position with no moving parts, and with a 0.5 aperture and a 20 square degree focal plane working in TDI (Time Delay Integration) mode. Such a large-scale facility would be uniquely capable for finding very large numbers of gamma ray bursts afterglow and supernovae ${ }^{39}$.

\section{CONCLUSION}

While Dome A could potentially be the best ground-based astronomical site for a wide range of observations, it is also the most remote and coldest location on the planet. Despite the difficulties associated with such a location, the PLATO automated site testing observatory was successfully deployed to Dome A in early 2008. It is still operational 5 months later and is collecting astronomical and atmospheric data. PLATO is an entirely self-powered observatory with a reliable multiply redundant hybrid power generation system. The command, control, and communications system for the observatory has proven robust against many potential failure points. The first-generation instrument suite described here is obtaining site testing data on the optical sky background, cloud cover statistics, the boundary layer turbulence, and the atmospheric opacity. There are future plans to extend this instrument suite to more fully characterise the site in the coming seasons. Additionally, moderate sized instruments are now acquiring datasets that should enable unique science in the field of transient and variable source astronomy. The results from these site testing and scientific instruments are crucial to quantify the Dome A atmospheric and environmental conditions required in order to plan for larger scale future astronomical facilities at this site.

\section{ACKNOWLEDGEMENTS}

The authors wish to thank all members of the 2008 Polar Research Institute of China Dome A expedition for a heroic effort in reaching the site and for providing invaluable assistance to the expedition astronomers in setting up the PLATO observatory. A number of staff and students from the University of New South Wales provided valuable "last minute" contributions that helped to ensure the success of this project: we particularly thank George Georgevits, Mikayla Keen, Tim Leslie, and Jessie Christiansen. This research is financially supported from the Australian Research Council, the Australian Antarctic Division, the Chinese Academy of Sciences, the National Natural Science Foundation of China, the US National Science Foundation, and the United States Antarctic Program. Additional financial contributions have been made by the majority of institutions involved in this collaboration. 


\section{REFERENCES}

[1] Storey, J.W.V., “Astronomy from Antarctica,” Antarctic Science 17, 555 (2005).

[2] Harper, D.A., "Infrared astronomy in Antarctica," Amer. Inst. Phys. Conf. Proc. 198, 123 (1989).

[3] Gillingham, P.R., "Prospects for an Antarctic Observatory," Proc. ASA 9, 55 (1991).

[4] Ashley, M.C.B., Burton, M.G., Storey, J.W.V., Lloyd, J.P., Bally, J., Briggs, J.W., Harper, D.A., "South Pole observations of the near-infrared sky brightness," PASP 108, 721 (1996).

[5] Nguyen, H.T., Rauscher, B.J., Harper, D.A., Loewenstein, R.F., Pernic, R.J., Severson, S.A., Hereld, M., "The South Pole Near Infrared Sky Brightness,” PASP 109, 718 (1996).

[6] Phillips, A., Burton, M.G., Ashley, M.C.B., Storey, J.W.V., Lloyd, J.P., Harper, D.A., Bally, J., "The near-infrared sky emission at the South Pole in winter," ApJ 527, 1009 (1999).

[7] Chamberlain, M.A., Ashley, M.C.B., Burton, M.G., Phillips, A., Storey, J.W.V., Harper, D.A., "Mid-infrared observing conditions at the South Pole," ApJ 535, 501 (2000).

[8] Chamberlin, R.A., "South Pole submillimeter sky opacity and correlations with radiosonde observations," J. Geophys. Res. 106, 20101 (2001).

[9] Loewenstein, R.F., Bero, C., Lloyd, J.P., Mrozek, F., Bally, J., Theil, D., “Astronomical Seeing at the South Pole," ASP Conf. Ser. 141, 296 (1998).

[10] Marks, R., Vernin, J., Azouit, M., Manigault, J.F., Clevelin, C., "Measurements of optical seeing on the high Antarctic plateau," A\&ASS 134, 161 (1999).

[11] Travouillon, T., Ashley, M.C.B., Burton, M.G., Storey, J.W.V., Loewenstein, R.F., "Atmospheric turbulence at the South Pole and its implications for astronomy," A\&A 400, 1163 (2003).

[12] Marks, R., "Astronomical seeing from the summits of the Antarctic plateau," A\&A 385, 328 (2002).

[13] Calisse, P.G., Ashley, M.C.B., Burton, M.G., Phillips, M.A., Storey, J.W.V., Radford, S.J.E., Peterson, J.B., "Submillimeter site testing at Dome C, Antarctica," PASA 21, 256 (2004).

[14] Walden, V.P., Town, M.S., Halter, B., Storey, J.W.V., "First measurements of the infrared sky brightness at Dome C, Antarctica," PASP 117, 300 (2005).

[15] Lawrence, J.S., Ashley, M.C.B., Tokovinin, A., Travouillon, T., "Exceptional astronomical seeing conditions above Dome C in Antarctica," Nature 431, 278 (2004).

[16] Agabi, K., Aristidi, E., Azouit, M., Fossat, E., Martin, F., Sadibekova, T., Vernin, J., Ziad, A., "First whole atmosphere nighttime seeing measurements at Dome C, Antarctica," PASP 118, 344 (2006).

[17] Trinquet, H., Agabi, A., Vernin, J., Azouit, M., Aristidi, E., Fossat, E., "Nighttime optical turbulence vertical structure above Dome C in Antarctica," PASP 120, 203 (2008).

[18] van Lipzig, N.P.M., Turner, J., Colwell, S.R., van Der Broeke, M.R., "The near-surface wind field over the Antarctic continent," Int. J. Climatology 24, 1973 (2004).

[19] Australian Antarctic Division, Australian Antarctic Data Centre website: http://www.aad.gov.au/weather/aws/domea/index.html (2008).

[20] Swain, M.R., Gallée, H., “Antarctic Boundary Layer Seeing,” PASP 118, 1190 (2006).

[21] Lawrence, J.S., "Infrared and sub-millimetre atmospheric characteristics of Antarctic high plateau sites," PASP 116, 482 (2004).

[22] Hengst, S., Allen, G.R., Ashley, M.C.B., Everett, J.R., Lawrence, J.S., Luong-Van, D., Storey, J.W.V., "PLATO Power - a robust, low environmental impact power generation system for the Antarctic plateau," Proc SPIE 7012, in press (2008). 
[23] Luong-Van, D.M., Ashley, M.C.B., Everett, J.R., Lawrence, J.S., Storey, J.W.V., "PLATO control and robotics," Proc SPIE 7019, in press (2008).

[24] Ashley, M.C.B., Burton, M.G., Lawrence, J.S., Storey, J.W.V., "Robotic telescopes on the Antarctic plateau," Astron. Nach. 325, 619 (2004).

[25] Strassmeier, K.G., Agabi, K., Agnoletto, L., et al., "Telescope and instrument robotization at Dome C," Astron Nach. 328, 451 (2007).

[26] Kulesa, C., et al., "Pre-HEAT: Submillimeter Site Testing and Astronomical Spectra from Dome A, Antarctica," Proc SPIE 7012, in press (2008).

[27] Bonner, C., et al., "SNODAR: a new instrument to measure the height of the atmospheric boundary layer on the Antarctic plateau," Proc SPIE 7014, in press (2008).

[28] Moore, A.M., Aristidi, E., Ashley, M.C.B., Candidi, M., Everett, J.R., Kenyon, S.L., Lawrence, J.S., Phillips, A., Le Roux, B., Ragazzoni, R., Salinari, P., Storey, J.W.V., Taylor, M., Travouillon, T., "The Gattini cameras for optical skybrightness measurements in Antarctica," Proc SPIE 6267, 62671N1 (2006).

[29] Skidmore, W., Travouillon, T., Riddle, R., "Evaluation of sonic anemometers as highly sensitive optical turbulence measuring devices for the Thirty Meter Telescope site testing campaign," Proc SPIE 6267, 62671Z (2006).

[30] Travouillon, T., Aristidi, E., Fossat, E., Lawrence, J., Mekarnia, D., Moore, A., Skidmore, W., Storey, J., "Sampling the Ground Layer of the Atmosphere at Dome C using fast Sonic-anemometers," Proc SPIE 7012, in press (2008).

[31] Kenyon, S.L., Ashley, M.C.B., Everett, J.R., Lawrence, J.S., Storey, J.W.V., "Nigel and the optical sky brightness at Dome C, Antarctica," Proc SPIE 6267, 6267M1 (2006).

[32] Dempsey, J.T., Storey, J.W.V., Phillips, M.A., "Auroral contribution to sky brightness for optical astronomy on the Antarctic plateau," PASA 22, 91 (2005).

[33] Hickson, P., Lanzetta, K., "Measuring Atmospheric Turbulence with a Lunar Scintillometer Array," PASP 116, 1143 (2004).

[34] Kornilov, V., Tokovinin, A., Vozyakova, O., Zaitsev, A., Shatsky, N., Potanin, S.F., Sarazin, M.S., "MASS: a monitor of the vertical turbulence distribution," Proc SPIE 4839, 837 (2003).

[35] Lawrence, J.S., Ashley, M.C.B., Lloyd, J.P., Tokovinin, A., Swain, M., Kenyon, S., Storey, J.W.V., “A robotic instrument for measuring high altitude atmospheric turbulence from Dome C, Antarctica," Proc SPIE 5489, 174 (2004).

[36] Durand, G., Cadelis, L., Minier, V., Veyssière, C., Walter, C., Pierre, A., Agabi, A., Fossat, E., Jeanneaux, F., “GIVRE: A Protection Against Frost Deposit on Polar Instruments," EAS Pub. Series 25, 77 (2007).

[37] Tosti, G., Busso, M., Nucciarelli, G., et al., "The International Robotic Antarctic Infrared Telescope (IRAIT),” Proc SPIE 6267, 62671H (2006).

[38] Walker, C.K., Kulesa, C.A., Golish, D.R., Hedden, A.S., Jacobs, K., Stutzki, J., Gao, J.R., Kooi, J., Glaister, D., Gully, W., Mehdi, I., Swain, M.R., Siegel, P., "Forecast for HEAT on Dome A, Antarctica: the High Elevation Antarctic Terahertz Telescope," Proc SPIE 5489, 470 (2004).

[39] York, D.G., Wang, L., Pennypacker, C., Cui, X., Cappellaro, E., Blouke, M., Lamb, D., Storey, J.W.V., Malina, R., Ashley, M.C.B., Basa, S., Zhou, X., Yuan, X., Harper, D., Sandford, D., Lawrence, J.S., Thorburn, J., "A large array of telescopes in Antarctica with all-sky imaging every 5 seconds," Proc SPIE 6267, 62671F1 (2006). 